\title{
Locally undetermined states, generalized Schmidt decomposition, and an application in distributed computing
}

\author{
Yuan Feng, Runyao Duan†, and Mingsheng Ying ${ }^{\ddagger}$ \\ Tsinghua National Laboratory for Information Science and Technology(TNList) \\ State Key Laboratory of Intelligent Technology and Systems, \\ Department of Computer Science and Technology, \\ Tsinghua University, Beijing, China.
}

March 21, 2019

\begin{abstract}
Multipartite quantum states that cannot be uniquely determined by their reduced states of all proper subsets of the parties exhibit some inherit 'high-order' correlation. This paper elaborates this issue by giving necessary and sufficient conditions for a pure multipartite state to be locally undetermined, and moreover, characterizing precisely all the pure states sharing the same set of reduced states with it. Interestingly, local determinability of pure states is closely related to a generalized notion of Schmidt decomposition. Furthermore, we find that locally undetermined states have some applications to the well-known consensus problem in distributed computation. To be specific, given some physically separated agents, when communication between them, either classical or quantum, is unreliable and they are not allowed to use local ancillary quantum systems, then there exists a totally correct and completely fault-tolerant protocol for them to reach a consensus if and only if they share a priori a locally undetermined quantum state.
\end{abstract}

\section{Introduction}

Entanglement is a striking feature of quantum mechanics which plays a central role in quantum computation and quantum information processing tasks such as quantum teleportation, superdense coding, and cryptographic protocols, etc [1]. In some sense, the advantage of quantum computation and quantum information processing over their classical counterparts is exactly due to the existence and proper use of entanglement. As a result, the theory of entanglement is important both theoretically and practically, and has been widely investigated in the past several decades.

Characterizing different types of entanglement is one of the most active research fields in entanglement theory. For multipartite states, one way towards such a characterization is to examine local determinability of them: if a quantum state shared among $n$ parties cannot be uniquely determined by its reduced states of fewer than $n$ parties, then in a sense the state exhibits 'higher-order' entanglement which is not attributable to all 'lower-order' entanglement among these parties. Surprisingly, Linden et al. 2, 3, 4, showed that in pure state case, chance for the existence of such 'higher-order' entanglement is very little. To be specific, almost all $n$-party pure states are determined by their reduced states of less than $n$ parties. In fact, when the number of parties is sufficiently large, for

\footnotetext{
*Email: feng-y@tsinghua.edu.cn

${ }^{\dagger}$ Email: dry@tsinghua.edu.cn

${ }^{\ddagger}$ Email: yingmsh@tsinghua.edu.cn
} 
almost all states except for a zero measure set, about two-thirds of the parties are sufficient to determine the global pure state. At the other extreme, Diósi [5] presented a method to construct a generic 3 -qubit pure state from its three 2 -qubit reduced states.

Although the set of locally undetermined pure states is proven to be zero measure, describing it precisely might be useful, as pointed out by Linden and Wootters [3], in investigating properties of multiparticle entanglement. Along this line, Walck and Lyons [6, 7] showed that in the special case of qubit systems, the only possible locally undetermined states are generalized GHZ-states. The main purpose of the current paper is to extend their result to the general case where Hilbert spaces with arbitrary dimensions are permitted. We present necessary and sufficient conditions for a multipartite pure state to be locally undetermined, and when a state is locally undetermined, we give the explicit form of all the pure states which share the same set of reduced states. Especially, we find that local determinability of pure states is closely related to a generalized notion of Schmidt decomposition which, to our best knowledge, is first defined in the present paper.

Distributed consensus is one of the central problems in distributed algorithms where a group of physically separated but inter-communicating agents need to reach agreement [8]. It has promising applications in distributed data processing and file management. In classical case, however, no deterministic protocol exists in an asynchronous setting which guarantees the correct agents to reach a consensus within finitely many steps, if some agents might fail during executing the protocol 9 . Even if probabilistic protocols are allowed, only one half of fail-stop faulty agents or one-third of malicious agents are tolerated if the probability of reaching agreement is required to be one [10.

D'Hondt and Panangaden first investigated distributed consensus with the aid of quantum resources [11. They proved that GHZ state, or GHZ-like states in higher dimensional case, is the only possible pure states to give a totally correct solution to the distributed consensus problem for an anonymous network in a purely quantum way. Here a protocol is called totally correct if it successfully terminates with its goal achieved within finitely many steps along each computation path, and it is purely quantum if no classical post-processing is allowed during the execution. The striking feature of GHZ-like states as quantum resource in solving distributed consensus is that they can not only solve the problem, but more importantly, the solution is fault-tolerant in the sense that no matter how unreliable the communication channels are - even if the communication, classical or quantum, is forbidden at all - and how many agents fail, the correct agents can still reach a consensus. We call this property completely fault-tolerant, which should be compared with the notion of fault-tolerance considered in [12] (which is usually assumed in classical setting) where faults are modeled by unpredictable behavior of some agents while the message exchange between agents is perfect. In this paper, we extend the result of D'Hondt and Panangaden by considering a more general network, anonymous or not, where a multipartite pure state is shared between the agents but any local ancillary quantum system is forbidden. Interestingly, we find that a totally correct and completely fault-tolerant protocol exists if and only if the shared state is locally undetermined.

\section{Generalized Schmidt decomposition of multipartite pure states}

This section is devoted to the definition of a generalized Schmidt decomposition of pure states in multipartite Hilbert space. Let $\rho$ be a density operator and $\rho=\sum_{i=1}^{M} \lambda_{i}|i\rangle\langle i|$ be its spectrum decomposition. Then $\operatorname{supp}(\rho)=\operatorname{span}\{|i\rangle: 1 \leq i \leq M\}$. For a set of density operators $\rho_{1}, \ldots, \rho_{n}$, we define $\operatorname{supp}\left\{\rho_{1}, \ldots, \rho_{n}\right\}=\sum_{i=1}^{n} \operatorname{supp}\left(\rho_{i}\right)$. Given a multipartite pure state $|\psi\rangle \in \mathcal{H}_{1} \otimes \cdots \otimes \mathcal{H}_{n}$

and $1 \leq k \leq n$, we denote by $\rho_{k}^{\psi}$ the 1-party reduced state of $|\psi\rangle$ on the $k$-th component subsystem, i.e., $\rho_{k}^{\psi}=\operatorname{Tr}_{\bar{k}}|\psi\rangle\langle\psi|$ where $\bar{k}$ indicates the Hilbert space $\bigotimes_{i \neq k} \mathcal{H}_{i} \equiv \mathcal{H}_{\bar{k}}$.

Definition 1 Let $|\psi\rangle \in \mathcal{H}_{1} \otimes \cdots \otimes \mathcal{H}_{n} . A$ family $\left\{P_{j}^{i}: i=1, \ldots, n ; j=1, \ldots, L\right\}$ of projectors are said to be Schmidt projectors of $|\psi\rangle$ if 


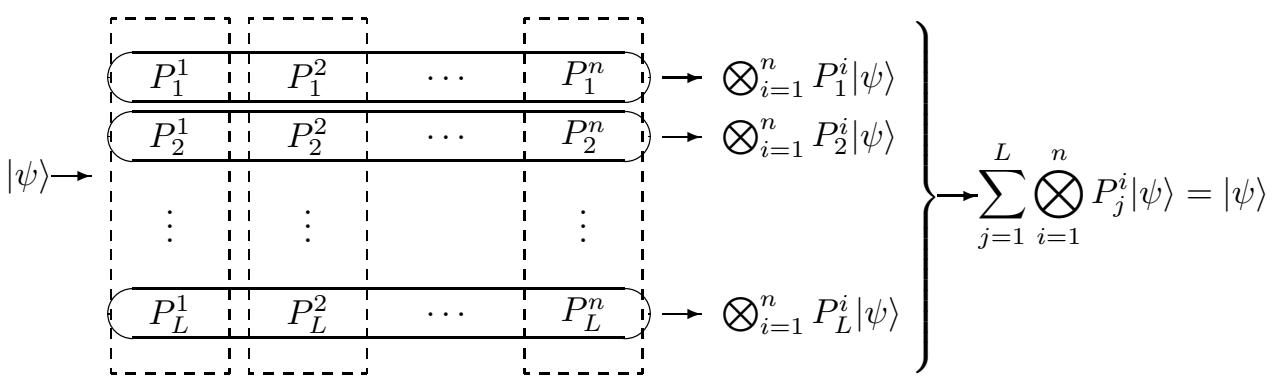

Figure 1: Illustration of the Schmidt projectors of a pure state $|\psi\rangle \in \mathcal{H}_{1} \otimes \cdots \otimes \mathcal{H}_{n}$. Here projectors in each column constitute an orthogonal set in the corresponding Hilbert space.

1. for any fixed $i, P_{j}^{i}: j=1, \ldots, L$ are pairwise orthogonal projectors on $\mathcal{H}_{i}$,

2. $P_{j}^{i}|\psi\rangle \neq 0$ for each $i$ and $j$,

3. $|\psi\rangle=\sum_{j=1}^{L} \bigotimes_{i=1}^{n} P_{j}^{i}|\psi\rangle$.

The projectors $P_{j}^{i}$ are illustrated in Fig. 1 .

The Schmidt number of $|\psi\rangle, S \operatorname{sch}(\psi)$, is defined to be the maximal $L$ such that $|\psi\rangle$ has $L$ rows of Schmidt projectors, that is

$$
\operatorname{Sch}(\psi)=\max \left\{L:|\psi\rangle \text { has Schmidt projectors } P_{j}^{i}, 1 \leq i \leq n, 1 \leq j \leq L\right\} .
$$

Note that every pure state has the trivial Schmidt projectors $\left\{I_{\mathcal{H}_{i}}: i=1, \ldots, n\right\}$ where $I_{\mathcal{H}_{i}}$ is the identity projector on $\mathcal{H}_{i}$. So the notion $\operatorname{Sch}(\psi)$ is well-defined. When $\operatorname{Sch}(\psi)>1,|\psi\rangle$ is said to be generalized Schmidt decomposable (GSD). If each Schmidt projector can be taken to be rank 1, then $|\psi\rangle$ is called completely GSD. It is well known that every bipartite pure state is completely GSD.

The next proposition indicates that we can put more constraints on the projectors which serve as the Schmidt projectors of a pure state. These constraints are useful in proving some later results in this paper.

Proposition 1 Let $|\psi\rangle \in \mathcal{H}_{1} \otimes \cdots \otimes \mathcal{H}_{n}$. Then $|\psi\rangle$ is GSD if and only if $|\psi\rangle$ has Schmidt operators $\left\{P_{j}^{i}: i=1, \ldots, n ; j=1, \ldots, L\right\}, L>1$, such that for any fixed $i,\left\{P_{j}^{i}: j=1, \ldots, L\right\}$ constitute $a$ projective measurement in $\operatorname{supp}\left(\rho_{i}^{\psi}\right)$, that is, $P_{j}^{i}$ are pairwise orthogonal and $\sum_{j=1}^{L} P_{j}^{i}=P_{\operatorname{supp}\left(\rho_{i}^{\psi}\right)}$. Here for a Hilbert space $\mathcal{H}$, we denote by $P_{\mathcal{H}}$ the projector onto $\mathcal{H}$.

Proof. Suppose we are given a set $\left\{P_{j}^{i}: i=1, \ldots, n ; j=1, \ldots, L\right\}$ where for each $i, P_{j}^{i}$ are pairwise orthogonal projectors on $\mathcal{H}_{i}, P_{j}^{i}|\psi\rangle \neq 0$, and $|\psi\rangle=\sum_{j=1}^{L} \bigotimes_{i=1}^{n} P_{j}^{i}|\psi\rangle$. Fix arbitrarily $1 \leq k \leq n$ and let $P=P_{\operatorname{supp}\left(\rho_{k}^{\psi}\right)}$. In the following we prove that $\left\{P P_{j}^{k} P: j=1, \ldots, L\right\}$ constitute a projective measurement in $\operatorname{supp}\left(\rho_{k}^{\psi}\right)$. To this end, we need to show: (i) $P P_{j}^{k} P$ is a nonnull projector for each j; (ii) $P P_{j}^{k} P$ are pairwise orthogonal; (iii) $\sum_{j=1}^{L} P P_{j}^{k} P=P$.

Take arbitrarily $1 \leq j \leq L$ and $1 \leq k^{\prime} \leq n$ such that $k^{\prime} \neq k$. Let $P_{\bar{j}}^{k^{\prime}}=\sum_{l \neq j} P_{l}^{k^{\prime}}$. It is easy to observe that

$$
P_{j}^{k}|\psi\rangle=\bigotimes_{i=1}^{n} P_{j}^{i}|\psi\rangle, \quad P_{\bar{j}}^{k^{\prime}}|\psi\rangle=\sum_{l \neq j} \bigotimes_{i=1}^{n} P_{l}^{i}|\psi\rangle .
$$

Hence

$$
P_{j}^{k}|\psi\rangle+P_{j}^{k^{\prime}}|\psi\rangle=|\psi\rangle
$$


Let

$$
|\psi\rangle=\sum_{i=1}^{M_{k}} \sqrt{\lambda_{i}}|i\rangle_{k}\left|\psi_{i}\right\rangle_{\bar{k}}, \quad \lambda_{1}, \ldots, \lambda_{M_{k}}>0
$$

be the (ordinary) Schmidt decomposition of $|\psi\rangle$ when treated as a bipartite state between $\mathcal{H}_{k}$ and $\mathcal{H}_{\bar{k}}$, where $M_{k} \leq d_{k}$, and $\left\{|i\rangle: i=1, \ldots, d_{k}\right\}$ is an orthonormal basis of $\mathcal{H}_{k}$. Then we have from Eq.(11)

$$
\sum_{i=1}^{M_{k}} \sqrt{\lambda_{i}}\left(P_{j}^{k}|i\rangle_{k}\right)\left|\psi_{i}\right\rangle_{\bar{k}}+\sum_{i=1}^{M_{k}} \sqrt{\lambda_{i}}|i\rangle_{k}\left(P_{\bar{j}}^{k^{\prime}}\left|\psi_{i}\right\rangle_{\bar{k}}\right)=\sum_{i=1}^{M_{k}} \sqrt{\lambda_{i}}|i\rangle_{k}\left|\psi_{i}\right\rangle_{\bar{k}}
$$

Multiplying both sides of the above equation by $\langle l|\left\langle\psi_{i}\right|$ for any $1 \leq i \leq M_{k}$ and $M_{k}<l \leq d_{k}$, we have $\left\langle l\left|P_{j}^{k}\right| i\right\rangle=0$. That is, for each $i=1, \ldots, M_{k}, P_{j}^{k}|i\rangle$ lies in the Hilbert space $\operatorname{supp}\left(\rho_{k}^{\psi}\right)=$ $\operatorname{span}\left\{|i\rangle: 1 \leq i \leq M_{k}\right\}$. As a consequence, we have $P P_{j}^{k}|i\rangle=P_{j}^{k}|i\rangle$, and then $P P_{j}^{k} P|\phi\rangle=P_{j}^{k} P|\phi\rangle$ for any $|\phi\rangle \in \mathcal{H}_{k}$. So $P P_{j}^{k} P=P_{j}^{k} P$. On the other hand, from the fact that $\sum_{j=1}^{L} P_{j}^{k}|\psi\rangle=|\psi\rangle$ and Eq.(2) we have $\sum_{j=1}^{L} P_{j}^{k}|i\rangle=|i\rangle$ for each $1 \leq i \leq M_{k}$. Thus $\sum_{j=1}^{L} P_{j}^{k} P=P$.

Now we can check (i), (ii), and (iii) directly. For (i), we notice that $P P_{j}^{k} P$ is positive, $P P_{j}^{k} P|\psi\rangle=$ $P_{j}^{k}|\psi\rangle \neq 0$, and

$$
\left(P P_{j}^{k} P\right)^{2}=P P_{j}^{k} P P_{j}^{k} P=P P_{j}^{k} P_{j}^{k} P=P P_{j}^{k} P .
$$

(ii) follows from $\left(P P_{j}^{k} P\right)\left(P P_{j^{\prime}}^{k} P\right)=P P_{j}^{k} P_{j^{\prime}}^{k} P=0$ when $j \neq j^{\prime}$, and (iii) from $\sum_{j=1}^{L} P P_{j}^{k} P=$ $\sum_{j=1}^{L} P_{j}^{k} P=P$.

\section{Characterization of locally undetermined states}

Given a multipartite quantum state $\rho$ in Hilbert space $\mathcal{H}_{1} \otimes \cdots \otimes \mathcal{H}_{n}$, we can easily determine its reduced state of any proper subset $S \subseteq\{1, \ldots, n\} \equiv \mathcal{N}$ by tracing out all the systems not included in $S$. The converse of this problem is, however, very complicated. On one hand, given states $\rho_{S_{1}}, \ldots, \rho_{S_{k}}$ for some proper subsets $S_{1}, \ldots, S_{k} \subseteq \mathcal{N}$, it is generally very difficult to determine if they are compatible in the sense that there exists some global state $\rho$ in $\mathcal{H}_{1} \otimes \cdots \otimes \mathcal{H}_{n}$ such that the reduced state of $\rho$ for the systems in $S_{i}$ is exactly $\rho_{S_{i}}$,13, 14, 15, 16, 17. On the other hand, even if the existence of such $\rho$ is pre-assumed, how to construct one, and furthermore, to characterize precisely all such states is still open. In the current paper, we only focus on local determinability of pure states among other pure states in the same Hilbert space. Allowing the considered states to be mixed will extremely increase the complexity of the problem. We leave this general case for further investigation.

Definition 2 Let $|\psi\rangle$ be a pure state in the composite Hilbert space $\mathcal{H}_{1} \otimes \cdots \otimes \mathcal{H}_{n}$ with $\operatorname{dim}\left(\mathcal{H}_{i}\right)=d_{i}$, $1 \leq i \leq n$. We say that $|\psi\rangle$ is locally undetermined (among pure states) if there exists a pure state $|\phi\rangle \in \mathcal{H}_{1} \otimes \cdots \otimes \mathcal{H}_{n}$ such that $|\langle\phi \mid \psi\rangle| \neq 1$, and $|\phi\rangle$ shares the same $n(n-1)$-party reduced states with $|\psi\rangle$, i.e.

$$
\operatorname{Tr}_{i}|\psi\rangle\left\langle\psi\left|=\operatorname{Tr}_{i}\right| \phi\right\rangle\langle\phi|: i=1, \ldots, n .
$$

It is worth noting that once the $n(n-1)$-party reduced states $\operatorname{Tr}_{i}|\psi\rangle\langle\psi|, 1 \leq i \leq n$, are specified, all $m$-party reduced states are specified as well for $m<n$. We denote by $R(\psi)$ the set of $|\phi\rangle$ satisfying Eq.(3). Then $|\psi\rangle$ is locally determined if and only if $|R(\psi)|=1$ where for a set $A,|A|$ is the cardinality of $A$. 
Theorem 1 A pure state $|\psi\rangle \in \mathcal{H}_{1} \otimes \cdots \otimes \mathcal{H}_{n}$ is locally undetermined if and only if it is GSD. Furthermore,

$$
R(\psi)=\left\{\sum_{j=1}^{L} \exp \left(1 \theta_{j}\right) \bigotimes_{i=1}^{n} P_{j}^{i}|\psi\rangle: P_{j}^{i} \text { are Schmidt projectors of }|\psi\rangle \text {, and } 0 \leq \theta_{1}, \ldots, \theta_{L}<2 \pi\right\} .
$$

Proof. For the sufficiency part, we need only prove that any state $|\phi\rangle$ in the set $R(\psi)$ defined in Eq.(4) shares the same set of $(n-1)$-party reduced states with $|\psi\rangle$. Let $|\phi\rangle=\sum_{j=1}^{L} \exp \left(1 \theta_{j}\right) \bigotimes_{i=1}^{n} P_{j}^{i}|\psi\rangle$ where $0 \leq \theta_{1}, \ldots, \theta_{L}<2 \pi$. Then for each $1 \leq k \leq n$, we have

$$
\begin{aligned}
\operatorname{Tr}_{k}|\phi\rangle\langle\phi| & =\operatorname{Tr}_{k}\left(\sum_{j, j^{\prime}=1}^{L} \exp \left(1\left(\theta_{j}-\theta_{j^{\prime}}\right)\right) \bigotimes_{i=1}^{n} P_{j}^{i}|\psi\rangle\langle\psi| \bigotimes_{i=1}^{n} P_{j^{\prime}}^{i}\right) \\
& =\sum_{j, j^{\prime}=1}^{L} \exp \left(1\left(\theta_{j}-\theta_{j^{\prime}}\right)\right) \bigotimes_{i \neq k} P_{j}^{i} \operatorname{Tr}_{k}\left(P_{j}^{k} P_{j^{\prime}}^{k}|\psi\rangle\langle\psi|\right) \bigotimes_{i \neq k} P_{j^{\prime}}^{i} \\
& =\sum_{j=1}^{L} \bigotimes_{i \neq k} P_{j}^{i} \operatorname{Tr}_{k}\left(P_{j}^{k} P_{j}^{k}|\psi\rangle\langle\psi|\right) \bigotimes_{i \neq k} P_{j}^{i} \\
& =\operatorname{Tr}_{k}\left(\sum_{j=1}^{L} \bigotimes_{i=1}^{n} P_{j}^{i}|\psi\rangle\langle\psi| \bigotimes_{i=1}^{n} P_{j}^{i}\right) .
\end{aligned}
$$

On the other hand, from Item 3 of Definition 1 we can easily check that the reduced state $\operatorname{Tr}_{k}|\psi\rangle\langle\psi|$ is exactly described by Eq.(8). That completes the proof of the sufficiency part.

Now we turn to the necessity part. The main proof technology is from Ref. [6]. Suppose $|R(\psi)|>$ 1. Let $|\phi\rangle \in R(\psi)$ but $|\langle\phi \mid \psi\rangle| \neq 1$. For any $1 \leq k \leq n$, since $\operatorname{Tr}_{k}|\psi\rangle\left\langle\psi\left|=\operatorname{Tr}_{k}\right| \phi\right\rangle\langle\phi|$, there exists unitary transformation $U_{k}$ on $\mathcal{H}_{k}$ such that $|\phi\rangle=U_{k}|\psi\rangle$. Let

$$
U_{k}=\sum_{i=1}^{d_{k}} \exp \left(\theta_{i}^{k}\right)|i\rangle_{k}\langle i|
$$

be the spectrum decomposition of $U_{k}$, where $0 \leq \theta_{i}^{k}<2 \pi$. Then $\left\{\left|i_{1}\right\rangle_{1} \ldots\left|i_{n}\right\rangle_{n}: i_{k}=1, \ldots, d_{k}, 1 \leq\right.$ $k \leq n\}$ constitute a complete orthonormal basis of $\mathcal{H}_{1} \otimes \cdots \otimes \mathcal{H}_{n}$. Write the decomposition of $|\psi\rangle$ under this basis as

$$
|\psi\rangle=\sum_{i_{1}=1}^{d_{1}} \cdots \sum_{i_{n}=1}^{d_{n}} C_{i_{1} \ldots i_{n}}\left|i_{1}\right\rangle_{1} \ldots\left|i_{n}\right\rangle_{n}=\sum_{I} C_{I}|I\rangle
$$

where $I=i_{1} \ldots i_{n}$. It is easy to check that for any $1 \leq k \leq n$ and $I$,

$$
\left(|i\rangle_{k}\langle i|\right)|I\rangle=\delta_{i, i_{k}}|I\rangle \text {. }
$$

Thus we have for any $j \neq k$,

$$
\begin{aligned}
U_{k}^{\dagger} \otimes U_{j}|\psi\rangle & =\sum_{I} C_{I} \sum_{i, i^{\prime}} \exp \left[1\left(\theta_{i}^{j}-\theta_{i^{\prime}}^{k}\right)\right]\left(|i\rangle_{j}\left\langle i|\otimes| i^{\prime}\right\rangle_{k}\left\langle i^{\prime}\right|\right)|I\rangle \\
& =\sum_{I} C_{I} \exp \left[1\left(\theta_{i_{j}}^{j}-\theta_{i_{k}}^{k}\right)\right]|I\rangle,
\end{aligned}
$$

and then $C_{I} \exp \left[1\left(\theta_{i_{j}}^{j}-\theta_{i_{k}}^{k}\right)\right]=C_{I}$ from the fact that $U_{k}^{\dagger} \otimes U_{j}|\psi\rangle=|\psi\rangle$. This means that whenever $C_{I} \neq 0$ in the decomposition Eq.(9) of $|\psi\rangle$, we have $\theta_{i_{j}}^{j}=\theta_{i_{k}}^{k}$ for any $j \neq k$. 
Denote by $\mathcal{I}$ the set of all the basis states $|I\rangle$ on which $|\psi\rangle$ has nonzero component, i.e.,

$$
\mathcal{I}=\left\{|I\rangle: C_{I}=\langle I \mid \psi\rangle \neq 0\right\}
$$

Then for any $|I\rangle,|J\rangle \in \mathcal{I}$, if $I$ and $J$ are adjacent, i.e., there exists $k_{0}$ such that $i_{k_{0}}=j_{k_{0}}$, then for any $1 \leq k \leq n$, we have

$$
\theta_{i_{k}}^{k}=\theta_{i_{k_{0}}}^{k_{0}}=\theta_{j_{k_{0}}}^{k_{0}}=\theta_{j_{k}}^{k} .
$$

Furthermore, if $I$ and $J$ are connected, i.e., there exists $\left|I^{1}\right\rangle, \ldots,\left|I^{m}\right\rangle \in \mathcal{I}$ such that $I^{1}=I, I^{m}=J$ and for each $1 \leq i \leq m-1, I^{i}$ and $I^{i+1}$ are adjacent, then from Eq.(10) we have

$$
\theta_{i_{k}}^{k}=\theta_{i_{k}^{1}}^{k}=\cdots=\theta_{i_{k}^{m}}^{k}=\theta_{j_{k}}^{k}
$$

for any $1 \leq k \leq n$.

Partition $\mathcal{I}$ into subsets $\mathcal{I}_{1}, \ldots, \mathcal{I}_{L}$ such that for any $|I\rangle,|J\rangle \in \mathcal{I},|I\rangle$ and $|J\rangle$ belong to a same $\mathcal{I}_{l}$ if and only if there are connected. According to this division, $|\psi\rangle$ can be rewritten as

$$
|\psi\rangle=\sum_{j=1}^{L} \sum_{|I\rangle \in \mathcal{I}_{j}} C_{I}|I\rangle
$$

We claim that $L>1$. Otherwise any $|I\rangle,|J\rangle$ in $\mathcal{I}$ are connected, hence from Eq.(11),

$$
|\phi\rangle=U_{k}|\psi\rangle=\sum_{i=1}^{d_{k}} \sum_{|I\rangle \in \mathcal{I}} C_{I}\left[\exp \left(1 \theta_{i}^{k}\right)|i\rangle_{k}\langle i|\right]|I\rangle=\sum_{|I\rangle \in \mathcal{I}} C_{I} \exp \left({ }_{1} \theta_{i_{k}}^{k}\right)|I\rangle=\exp \left({ }_{1} \theta_{k}\right)|\psi\rangle,
$$

a contradiction.

Now for each $1 \leq k \leq n$ and $1 \leq j \leq L$, let

$$
A_{j}^{k}=\left\{\left|i_{k}\right\rangle_{k} \text { : there exist }\left|i_{j}\right\rangle_{j}, 1 \leq j \leq n \text { but } j \neq k \text {, such that }\left|i_{1}\right\rangle \ldots\left|i_{n}\right\rangle \in \mathcal{I}_{j}\right\},
$$

and $P_{j}^{k}=\sum_{\left|i_{k}\right\rangle \in A_{j}^{k}}\left|i_{k}\right\rangle\left\langle i_{k}\right|$ be a projector on $\mathcal{H}_{k}$. Take arbitrarily $\left|l_{k}\right\rangle \in A_{j}^{k}$ and $\left|l_{k}^{\prime}\right\rangle \in A_{j^{\prime}}^{k}$ for $j \neq j^{\prime}$. By definition, there exist $|I\rangle \in \mathcal{I}_{j}$ and $\left|I^{\prime}\right\rangle \in \mathcal{I}_{j^{\prime}}$ such that $i_{k}=l_{k}$ and $i_{k}^{\prime}=l_{k}^{\prime}$. Since $I$ and $I^{\prime}$ are not adjacent (otherwise $j=j^{\prime}$ ), we have $i_{k} \neq i_{k}^{\prime}$, and hence $\left\langle l_{k} \mid l_{k}^{\prime}\right\rangle=\left\langle i_{k} \mid i_{k}^{\prime}\right\rangle=0$. That is, the projectors $P_{j}^{k}: j=1, \ldots, L$ are pairwise orthogonal for any fixed $k$.

If $|I\rangle \in \mathcal{I}_{j}$, then $i_{k} \in A_{j}^{k}$ and hence $P_{j}^{k}|I\rangle=|I\rangle$. If $|I\rangle \notin \mathcal{I}_{j}$, then by definition, for any $J \in \mathcal{I}_{j}$, $i_{k} \neq j_{k}$. So $i_{k} \notin A_{j}^{k}$ and $P_{j}^{k}|I\rangle=0$. In a word, for any $|I\rangle \in \mathcal{I}$ and $1 \leq k \leq n$,

$$
P_{j}^{k}|I\rangle= \begin{cases}|I\rangle, & \text { if }|I\rangle \in \mathcal{I}_{j} \\ 0, & \text { if }|I\rangle \notin \mathcal{I}_{j}\end{cases}
$$

We derive further that for any $j=1, \ldots, n$,

$$
\bigotimes_{i=1}^{n} P_{j}^{i}|\psi\rangle=\sum_{j^{\prime}=1}^{L} \sum_{|I\rangle \in \mathcal{I}_{j^{\prime}}} C_{I} \bigotimes_{i=1}^{n} P_{j}^{i}|I\rangle=\sum_{|I\rangle \in \mathcal{I}_{j}} C_{I}|I\rangle
$$

and hence $|\psi\rangle$ is GSD.

Finally, let $R=\left\{\sum_{j=1}^{L} \exp \left({ }_{1} \theta_{j}\right) \bigotimes_{i=1}^{n} P_{j}^{i}|\psi\rangle: 0 \leq \theta_{1}, \ldots, \theta_{L}<2 \pi\right\}$. We need to show $R(\psi)=R$ to finish the proof of this theorem. Note that at the sufficiency part, we have already proved 
$R \subseteq R(\psi)$. To show the opposite side, let $|\phi\rangle \in R(\psi)$ and fix arbitrarily $k$. Then

$$
\begin{aligned}
|\phi\rangle=U_{k}|\psi\rangle & =\sum_{i=1}^{d_{k}} \sum_{j=1}^{L} \sum_{|I\rangle \in \mathcal{I}_{j}} C_{I}\left[\exp \left(1 \theta_{i}^{k}\right)|i\rangle_{k}\langle i|\right]|I\rangle \\
& =\sum_{j=1}^{L} \sum_{|I\rangle \in \mathcal{I}_{j}} C_{I} \exp \left(1 \theta_{i_{k}}^{k}\right)|I\rangle \\
& =\sum_{j=1}^{L} \exp \left(1 \theta_{j}\right) \sum_{|I\rangle \in \mathcal{I}_{j}} C_{I}|I\rangle \\
& =\sum_{j=1}^{L} \exp \left(1 \theta_{j}\right) \bigotimes_{i=1}^{n} P_{j}^{i}|\psi\rangle,
\end{aligned}
$$

where the fourth equation follows from Eq.(11) and the last from Eq.(13).

If we are not concerned with the set $R(\psi)$, a simpler criteria for local determinability can be derived, as the following corollary states.

Corollary 1 Pure state $|\psi\rangle \in \mathcal{H}_{1} \otimes \cdots \otimes \mathcal{H}_{n}$ is locally undetermined if and only if for each $i=$ $1, \ldots, n$, there exist projectors $P_{1}^{i}$ and $P_{2}^{i}$ satisfying $P_{1}^{i}|\psi\rangle \neq 0, P_{2}^{i}|\psi\rangle \neq 0$, and $P_{1}^{i} \perp P_{2}^{i}$, such that

$$
|\psi\rangle=\bigotimes_{i=1}^{n} P_{1}^{i}|\psi\rangle+\bigotimes_{i=1}^{n} P_{2}^{i}|\psi\rangle
$$

Proof. The sufficiency part is direct from Theorem 1 For the necessity part, suppose $|\psi\rangle$ is locally undetermined. Then from Theorem 1, projectors $\left\{Q_{j}^{i}: i=1, \ldots, n ; j=1, \ldots, L\right\}, L \geq 2$, can be found such that for any $i,\left\{Q_{j}^{i}: j=1, \ldots, L\right\}$ are pairwise orthogonal in $\mathcal{H}_{i}, Q_{j}^{i}|\psi\rangle \neq 0$, and

$$
|\psi\rangle=\sum_{j=1}^{L} \bigotimes_{i=1}^{n} Q_{j}^{i}|\psi\rangle
$$

Let $P_{1}^{i}=Q_{1}^{i}$ and $P_{2}^{i}=\sum_{j=2}^{L} Q_{j}^{i}$. Then $P_{1}^{i} \perp P_{2}^{i}, P_{1}^{i}|\psi\rangle \neq 0$, and

$$
\begin{aligned}
\bigotimes_{i=1}^{n} P_{2}^{i}|\psi\rangle & =\left[\sum_{j_{1}, \ldots, j_{n}=2}^{L} \bigotimes_{i=1}^{n} Q_{j_{i}}^{i}\right] \sum_{j=1}^{L} \bigotimes_{i=1}^{n} Q_{j}^{i}|\psi\rangle \\
& =\sum_{j=1}^{L} \sum_{j_{1}, \ldots, j_{n}=2}^{L} \delta_{j, j_{1}} \cdots \delta_{j, j_{n}} \bigotimes_{i=1}^{n} Q_{j}^{i}|\psi\rangle \\
& =\sum_{j=2}^{L} \bigotimes_{i=1}^{n} Q_{j}^{i}|\psi\rangle .
\end{aligned}
$$

Hence we have

$$
\bigotimes_{i=1}^{n} P_{1}^{i}|\psi\rangle+\bigotimes_{i=1}^{n} P_{2}^{i}|\psi\rangle=\bigotimes_{i=1}^{n} Q_{1}^{i}|\psi\rangle+\sum_{j=2}^{L} \bigotimes_{i=1}^{n} Q_{j}^{i}|\psi\rangle=|\psi\rangle .
$$

Now we show that $P_{2}^{i}|\psi\rangle \neq 0$ for any $i$. Otherwise $\bigotimes_{i=1}^{n} P_{2}^{i}|\psi\rangle=0$, and then $|\psi\rangle=\bigotimes_{i=1}^{n} P_{1}^{i}|\psi\rangle$. So we derive that $Q_{j}^{i}|\psi\rangle=0$ for any $j>1$, which is a contradiction. 
Theorem 1 provides a necessary and sufficient condition for a pure multipartite state to be locally undetermined by means of generalized Schmidt decomposability. The Schmidt projectors are, however, hard to find in general. In the next theorem, by employing (ordinary bipartite) Schmidt decomposition for some proper partition of the original parties, we obtain a more practical method to determine the local determinability of a pure state.

Theorem 2 Let $|\psi\rangle$ be a pure state in $\mathcal{H}_{1} \otimes \cdots \otimes \mathcal{H}_{n}$. If $|\psi\rangle$ is locally undetermined, then for any $1 \leq k \leq n$ there exists a (ordinary) Schmidt decomposition

$$
|\psi\rangle=\sum_{i=1}^{M_{k}} \sqrt{\lambda_{i}}|i\rangle_{k}\left|\psi_{i}\right\rangle_{\bar{k}}, \quad \lambda_{1}, \ldots, \lambda_{M_{k}}>0
$$

of $|\psi\rangle$ when treated as a bipartite state between $\mathcal{H}_{k}$ and $\mathcal{H}_{\bar{k}}$, and a complete partition $S_{1}, \ldots, S_{L}$, $L \geq 2$, of $\left\{1, \ldots, M_{k}\right\} \equiv \mathcal{M}_{k}$ such that for any $j \neq k, 1 \leq l \neq l^{\prime} \leq L, r \in S_{l}, t \in S_{l^{\prime}}$, it holds that

$$
\rho_{j}^{\psi_{r}} \perp \rho_{j}^{\psi_{t}} .
$$

Furthermore

$R(\psi)=\left\{\sum_{j=1}^{L} \exp \left(1 \theta_{j}\right) \sum_{i \in S_{j}} \sqrt{\lambda_{i}}|i\rangle_{k}\left|\psi_{i}\right\rangle_{\bar{k}}: S_{j}\right.$ satisfy the conditions above, and $\left.0 \leq \theta_{1}, \ldots, \theta_{L}<2 \pi\right\}$.

Conversely, if there exists $1 \leq k \leq n$ such that a Schmidt decomposition of $|\psi\rangle$ and a partition of $\mathcal{M}_{k}$ satisfying the conditions presented above can be found, then $|\psi\rangle$ is locally undetermined.

Proof. Suppose $|\psi\rangle$ is locally undetermined. Then from Theorem 1 and Proposition 1 , there exist $\left\{P_{j}^{i}: i=1, \ldots, n ; j=1, \ldots, L\right\}, L>1$, such that for any $i, P_{j}^{i}: j=1, \ldots, L$ constitute a projective measurement in $\operatorname{supp}\left(\rho_{i}^{\psi}\right)$, and $|\psi\rangle=\sum_{j=1}^{L} \bigotimes_{i=1}^{n} P_{j}^{i}|\psi\rangle$.

For any $1 \leq k \leq n$ and $1 \leq j \leq L$, let

$$
\bigotimes_{i=1}^{n} P_{j}^{i}|\psi\rangle=\sum_{i \in S_{j}} \sqrt{\lambda_{i}}|i\rangle_{k}\left|\psi_{i}\right\rangle_{\bar{k}}
$$

be a Schmidt decomposition of the unnormalized state $\bigotimes_{i=1}^{n} P_{j}^{i}|\psi\rangle$ when treated as a bipartite state between $\mathcal{H}_{k}$ and $\mathcal{H}_{\bar{k}}$, where for each $i \in S_{j}, \lambda_{i}>0$, and $|i\rangle$ and $\left|\psi_{i}\right\rangle$ are normalized. It is easy to check that $P_{j}^{k}|i\rangle_{k}=|i\rangle_{k}$ and $P_{j}^{k^{\prime}}\left|\psi_{i}\right\rangle_{\bar{k}}=\left|\psi_{i}\right\rangle_{\bar{k}}$ for any $k^{\prime} \neq k$ and $i \in S_{j}$.

For any $j \neq j^{\prime}, i \in S_{j}$, and $i^{\prime} \in S_{j^{\prime}}$, we have ${ }_{k}\left\langle i \mid i^{\prime}\right\rangle_{k}={ }_{k}\left\langle i\left|P_{j}^{k} P_{j^{\prime}}^{k}\right| i^{\prime}\right\rangle_{k}=0$ since $P_{j}^{k} \perp P_{j^{\prime}}^{k}$, and ${ }_{\bar{k}}\left\langle\psi_{i} \mid \psi_{i^{\prime}}\right\rangle_{\bar{k}}={ }_{\bar{k}}\left\langle\psi_{i}\left|P_{j}^{k^{\prime}} P_{j^{\prime}}^{k^{\prime}}\right| \psi_{i^{\prime}}\right\rangle_{\bar{k}}=0$ since $P_{j}^{k^{\prime}} \perp P_{j^{\prime}}^{k^{\prime}}$. As a consequence,

$$
|\psi\rangle=\sum_{j=1}^{L} \bigotimes_{i=1}^{n} P_{j}^{i}|\psi\rangle=\sum_{j=1}^{L} \sum_{i \in S_{j}} \sqrt{\lambda_{i}}|i\rangle_{k}\left|\psi_{i}\right\rangle_{\bar{k}}
$$

is a Schmidt decomposition of $|\psi\rangle$.

For any $j \neq k, 1 \leq l \neq l^{\prime} \leq L, r \in S_{l}$, and $t \in S_{l^{\prime}}$, we have

$$
\rho_{j}^{\psi_{r}}=\operatorname{Tr}_{\bar{j}}\left|\psi_{r}\right\rangle_{\bar{k}}\left\langle\psi_{r}\right|=\operatorname{Tr}_{\bar{j}}\left[P_{l}^{j}\left|\psi_{r}\right\rangle_{\bar{k}}\left\langle\psi_{r}\right| P_{l}^{j}\right]=P_{l}^{j}\left[\operatorname{Tr}_{\bar{j}}\left|\psi_{r}\right\rangle_{\bar{k}}\left\langle\psi_{r}\right|\right] P_{l}^{j}
$$

and

$$
\rho_{j}^{\psi_{t}}=\operatorname{Tr}_{\bar{j}}\left|\psi_{t}\right\rangle_{\bar{k}}\left\langle\psi_{t}\right|=\operatorname{Tr}_{\bar{j}}\left[P_{l^{\prime}}^{j}\left|\psi_{s}\right\rangle_{\bar{k}}\left\langle\psi_{s}\right| P_{l^{\prime}}^{j}\right]=P_{l^{\prime}}^{j}\left[\operatorname{Tr}_{\bar{j}}\left|\psi_{s}\right\rangle_{\bar{k}}\left\langle\psi_{s}\right|\right] P_{l^{\prime}}^{j}
$$


So $\rho_{j}^{\psi_{r}} \perp \rho_{j}^{\psi_{t}}$ from the orthogonality of $P_{l}^{j}$ and $P_{l^{\prime}}^{j}$.

Furthermore, from Eqs.(44) and (18) we derive that

$$
\begin{aligned}
R(\psi) & =\sum_{j=1}^{L} \exp \left(1 \theta_{j}\right) \bigotimes_{i=1}^{n} P_{j}^{i}|\psi\rangle \\
& =\sum_{j=1}^{L} \exp \left(1 \theta_{j}\right) \sum_{i \in S_{j}} \sqrt{\lambda_{i}}|i\rangle_{k}\left|\psi_{i}\right\rangle_{\bar{k}}
\end{aligned}
$$

Conversely, suppose there exists $1 \leq k \leq n$ such that a Schmidt decomposition of $|\psi\rangle$ and a partition of $\mathcal{M}_{k}$ satisfying the conditions presented in the Theorem can be found. For any $j \neq k$ and $1 \leq l \leq L$, let $\mathcal{H}_{l}^{j}=\operatorname{supp}\left\{\rho_{j}^{\psi_{i}}: i \in S_{l}\right\}$, and $P_{l}^{j}$ be the projector onto $\mathcal{H}_{l}^{j}$. Let $P_{l}^{k}=\sum_{i \in S_{l}}|i\rangle_{k}\langle i|$. Then it is obvious that for any $1 \leq j \leq n, P_{l}^{j}$ are pairwise orthogonal projectors on $\mathcal{H}_{j}$, and $P_{l}^{j}|\psi\rangle \neq 0$. Furthermore, for any $j \neq k, 1 \leq i \leq M_{k}, 1 \leq l \leq L$, we have $P_{l}^{k}|i\rangle_{k}=\delta_{i \in S_{l}}|i\rangle_{k}$ $P_{l}^{j}\left|\psi_{i}\right\rangle_{\bar{k}}=\delta_{i \in S_{l}}\left|\psi_{i}\right\rangle_{\bar{k}}$, where $\delta_{i \in A}$ equals 1 if $i \in A$ while 0 if $i \notin A$. Hence we deduce that

$$
\begin{aligned}
\sum_{l=1}^{L} \bigotimes_{j=1}^{n} P_{l}^{j}|\psi\rangle & =\sum_{l=1}^{L} \bigotimes_{j=1}^{n} P_{l}^{j}\left[\sum_{i=1}^{M_{k}} \sqrt{\lambda_{i}}|i\rangle_{k}\left|\psi_{i}\right\rangle_{\bar{k}}\right] \\
& =\sum_{l=1}^{L} \sum_{i=1}^{M_{k}} \sqrt{\lambda_{i}}\left[P_{l}^{k}|i\rangle_{k}\right]\left[\bigotimes_{j \neq k} P_{l}^{j}\left|\psi_{i}\right\rangle_{\bar{k}}\right] \\
& =\sum_{l=1}^{L} \sum_{i \in S_{l}} \sqrt{\lambda_{i}}|i\rangle_{k}\left|\psi_{i}\right\rangle_{\bar{k}} \\
& =|\psi\rangle .
\end{aligned}
$$

Then $|\psi\rangle$ is locally undetermined from Theorem 1 .

Following Theorem 2 we can obtain a simple way to check whether $|\psi\rangle$ is locally undetermined when one of the 1-party reduced states has distinct nonzero eigenvalues.

Corollary 2 Suppose $|\psi\rangle \in \mathcal{H}_{1} \otimes \cdots \otimes \mathcal{H}_{n}$ and there exists $1 \leq k \leq n$ such that $\rho_{k}^{\psi}$ has distinct nonzero eigenvalues, and suppose the Schmidt decomposition of $|\psi\rangle$ when treated as a bipartite state between $\mathcal{H}_{k}$ and $\mathcal{H}_{\bar{k}}$ has the form

$$
|\psi\rangle=\sum_{i=1}^{M_{k}} \sqrt{\lambda_{i}}|i\rangle_{k}\left|\psi_{i}\right\rangle_{\bar{k}}
$$

where $\lambda_{1}>\cdots>\lambda_{M_{k}}$. Then $|\psi\rangle$ is locally undetermined if and only if there exists a complete partition $S_{1}, \ldots, S_{L}$ of $\left\{1, \ldots, M_{k}\right\} \equiv \mathcal{M}_{k}$ such that for any $j \neq k, 1 \leq l \neq l^{\prime} \leq L, r \in S_{l}, t \in S_{l^{\prime}}$, it holds that

$$
\rho_{j}^{\psi_{r}} \perp \rho_{j}^{\psi_{t}} .
$$

Furthermore

$R(\psi)=\left\{\sum_{j=1}^{L} \exp \left(1 \theta_{j}\right) \sum_{i \in S_{j}} \sqrt{\lambda_{i}}|i\rangle_{k}\left|\psi_{i}\right\rangle_{\bar{k}}: S_{j}\right.$ satisfy the conditions above, and $\left.0 \leq \theta_{1}, \ldots, \theta_{L}<2 \pi\right\}$.

Particularly, if $M_{k}=2$, then $|\psi\rangle$ is locally undetermined if and only if for any $j \neq k$,

$$
\rho_{j}^{\psi_{1}} \perp \rho_{j}^{\psi_{2}}
$$


and

$$
R(\psi)=\left\{\sqrt{\lambda_{1}}|1\rangle_{k}\left|\psi_{1}\right\rangle_{\bar{k}}+\exp (1 \theta) \sqrt{\lambda_{2}}|2\rangle_{k}\left|\psi_{2}\right\rangle_{\bar{k}}: 0 \leq \theta<2 \pi\right\} .
$$

Proof. Notice that when $\rho_{k}^{\psi}$ has distinct nonzero eigenvalues, the Schmidt decomposition of $|\psi\rangle$ under the partition $\{k, \bar{k}\}$ of $\{1, \ldots, n\}$ has a unique form as in Eq.(20). Then the corollary follows directly from Theorem 2 .

Theorem 3 Suppose $|\psi\rangle \in \mathcal{H}_{1} \otimes \cdots \otimes \mathcal{H}_{n}$ for $n \geq 3$ and

$$
|\psi\rangle=\sum_{i=1}^{m} \sqrt{\lambda_{i}}|i\rangle_{1} \ldots|i\rangle_{n}
$$

is completely GSD where $m \leq \min \left\{d_{k}: 1 \leq k \leq n\right\}, \lambda_{1}, \ldots, \lambda_{m}>0$, and $\left\{|i\rangle_{k}: i=1, \ldots, d_{k}\right\}$ is an orthonormal basis for each $\mathcal{H}_{k}$. Then $|\psi\rangle$ is locally undetermined if and only if $m>1$, and when $m>1$,

$$
R(\psi)=\left\{\sum_{i=1}^{m} \sqrt{\lambda_{i}} \exp \left(1 \theta_{i}\right)|i\rangle_{1} \ldots|i\rangle_{n}: 0 \leq \theta_{1}, \ldots, \theta_{m}<2 \pi\right\} .
$$

Proof. First it is easy to check that $|\psi\rangle$ is locally undetermined if and only if $m>1$. Suppose $m>1$. Then from Theorem 1, any $|\phi\rangle \in R(\psi)$ has the form $|\phi\rangle=\sum_{j=1}^{L} \exp \left(1 \theta_{j}\right) \bigotimes_{i=1}^{n} P_{j}^{i}|\psi\rangle$ where $L>1$, $0 \leq \theta_{1}, \ldots, \theta_{L} \leq 2 \pi, P_{j}^{i}: j=1, \ldots, L$ are pairwise orthogonal projectors on $\mathcal{H}_{i}$, and

$$
|\psi\rangle=\sum_{j=1}^{L} \bigotimes_{i=1}^{n} P_{j}^{i}|\psi\rangle
$$

Fix arbitrarily $1 \leq j \leq L$. For any $1 \leq i, i^{\prime} \leq n$, we observe that

$$
P_{j}^{i}|\psi\rangle=P_{j}^{i^{\prime}}|\psi\rangle=\bigotimes_{i=1}^{n} P_{j}^{i}|\psi\rangle
$$

hence from Eq.(24)

$$
\sum_{l=1}^{m} \sqrt{\lambda_{l}}|l\rangle_{1} \ldots\left[P_{j}^{i}|l\rangle_{i}\right] \ldots|l\rangle_{n}=\sum_{l=1}^{m} \sqrt{\lambda_{l}}|l\rangle_{1} \ldots\left[P_{j}^{i^{\prime}}|l\rangle_{i^{\prime}}\right] \ldots|l\rangle_{n},
$$

and ${ }_{i}\left\langle l\left|P_{j}^{i}\right| l\right\rangle_{i}={ }_{i^{\prime}}\left\langle l\left|P_{j}^{i^{\prime}}\right| l\right\rangle_{i^{\prime}}$ by multiplying both sides by ${ }_{1}\langle l| \ldots{ }_{n}\langle l|$. That is, the quantity ${ }_{i}\left\langle l\left|P_{j}^{i}\right| l\right\rangle_{i}$ is independent of $i$. Let $\alpha_{j, l}={ }_{i}\left\langle l\left|P_{j}^{i}\right| l\right\rangle_{i} \geq 0$. Then from Eq.(26) we have

$$
P_{j}^{i}|l\rangle_{i}=\alpha_{j, l}|l\rangle_{i}
$$

Furthermore, from the relation

$$
\sum_{j=1}^{L} P_{j}^{i}|\psi\rangle=\sum_{j=1}^{L} \bigotimes_{i=1}^{n} P_{j}^{i}|\psi\rangle=|\psi\rangle
$$

we can deduce that $\sum_{j=1}^{L} \alpha_{j, l}=1$ for each $1 \leq l \leq m$. On the other hand, taking Eq.(27) back into Eq.(25) we have

$$
\sum_{l=1}^{m} \sqrt{\lambda_{l}}|l\rangle_{1} \ldots|l\rangle_{n}=\sum_{l=1}^{m} \sum_{j=1}^{L} \sqrt{\lambda_{l}} \bigotimes_{i=1}^{n}\left(P_{j}^{i}|l\rangle_{i}\right)=\sum_{l=1}^{m} \sum_{j=1}^{L} \sqrt{\lambda_{l}} \alpha_{j, l}^{n}|l\rangle_{1} \ldots|l\rangle_{n} .
$$


So $\sum_{j=1}^{L} \alpha_{j, l}^{n}=1$, and hence for each $1 \leq l \leq m$, there exists one and only one $j$, denoted by $j_{l}$ such that $\alpha_{j, l}=1$; other $\alpha_{j, l}$ equal 0 . Now we can calculate that

$$
\begin{aligned}
|\phi\rangle & =\sum_{j=1}^{L} \exp \left(1 \theta_{j}\right) \bigotimes_{i=1}^{n} P_{j}^{i}|\psi\rangle \\
& =\sum_{j=1}^{L} \exp \left(1 \theta_{j}\right) \sum_{l=1}^{m} \sqrt{\lambda_{l}} \alpha_{j, l}^{n}|l\rangle_{1} \ldots|l\rangle_{n} \\
& =\sum_{l=1}^{m} \sqrt{\lambda_{l}} \exp \left(1 \theta_{j_{l}}\right)|l\rangle_{1} \ldots|l\rangle_{n} .
\end{aligned}
$$

That completes the proof of the theorem.

Corollary 3 Suppose $|\psi\rangle$ is a pure state in n-qubit system, i.e., $\operatorname{dim}\left(\mathcal{H}_{i}\right)=2$ for each $1 \leq i \leq n$. Then $|\psi\rangle$ is locally undetermined if and only if $|\psi\rangle$ is completely GSD (or, as stated in [6], $|\psi\rangle$ is a generalized GHZ state): $|\psi\rangle=\alpha|0\rangle_{1} \ldots|0\rangle_{n}+\beta|1\rangle_{1} \ldots|1\rangle_{n}$ with $\alpha>0$ and $\beta>0$. Furthermore, if $|\psi\rangle$ is locally undetermined, then

$$
R(\psi)=\left\{\text { all maximally entangled states in } \mathbb{C}^{2} \otimes \mathbb{C}^{2} \text { space }\right\}
$$

when $n=2$ and $\alpha=\beta$; otherwise

$$
R(\psi)=\left\{\alpha|0\rangle_{1} \ldots|0\rangle_{n}+\exp (1 \theta) \beta|1\rangle_{1} \ldots|1\rangle_{n}: 0 \leq \theta<2 \pi\right\} .
$$

Proof. From Theorem $1 \psi\rangle\rangle$ is locally undetermined if and only if there exists an orthonormal basis, denoted by $\left\{|\widehat{0}\rangle_{i},|\widehat{1}\rangle_{i}\right\}$, for each $\mathcal{H}_{i}$ such that

$$
\begin{aligned}
|\psi\rangle & =\left(\bigotimes_{i=1}^{n}|\widehat{0}\rangle_{i}\langle\widehat{0}|\right)|\psi\rangle+\left(\bigotimes_{i=1}^{n}|\widehat{1}\rangle_{i}\langle\widehat{1}|\right)|\psi\rangle \\
& =\widehat{\alpha}|\widehat{0}\rangle_{1} \ldots|\widehat{0}\rangle_{n}+\widehat{\beta}|\widehat{1}\rangle_{1} \ldots|\widehat{1}\rangle_{n},
\end{aligned}
$$

where $\widehat{\alpha}={ }_{1}\langle\widehat{0}| \ldots{ }_{n}\langle\widehat{0} \mid \psi\rangle$ and $\widehat{\beta}={ }_{1}\langle\widehat{1}| \ldots{ }_{n}\langle\widehat{1} \mid \psi\rangle$. From the fact that $|\widehat{0}\rangle_{i}\langle\widehat{0} \mid \psi\rangle \neq 0$ for each $i$, we know $\widehat{\alpha} \neq 0$. Similarly, it holds that $\widehat{\beta} \neq 0$. Let $\widehat{\alpha}=\alpha \exp \left(1 \theta_{\alpha}\right)$ and $\widehat{\beta}=\beta \exp \left(1 \theta_{\beta}\right)$ where $\alpha=|\widehat{\alpha}|>0$ and $\beta=|\widehat{\beta}|>0$. Then we have $|\psi\rangle=\alpha|0\rangle_{1} \ldots|0\rangle_{n}+\beta|1\rangle_{1} \ldots|1\rangle_{n}$ by, say, letting $|0\rangle_{1}=\exp \left(1 \theta_{\alpha}\right)|\widehat{0}\rangle_{1}$, $|1\rangle_{1}=\exp \left(1 \theta_{\beta}\right)|\widehat{1}\rangle_{1}$, and $|0\rangle_{i}=|\widehat{0}\rangle_{i}$ and $|1\rangle_{i}=|\widehat{1}\rangle_{i}$ for $i \geq 2$.

When $n=2$ and $\alpha=\beta$, we have $\rho_{1}^{\psi}=\rho_{2}^{\psi}=I / 2$. Hence $|\phi\rangle \in R(\psi)$ if and only if $|\phi\rangle$ is a maximally entangled states in $\mathbb{C}^{2} \otimes \mathbb{C}^{2}$. Furthermore, we can show that $R(\psi)$ has the form in Eq.(28) by Corollary 2 for the case of $n=2$ and $\alpha \neq \beta$ while by Theorem 3 for the case of $n \geq 3$.

To conclude this section, we would like to point out that the techniques developed in this section can be used in locally determining an $n$-party pure state when only a proper subset of the $(n-1)$ party reduced states are specified. To be specific, we call a pure state $|\psi\rangle \in \mathcal{H}_{1} \otimes \cdots \otimes \mathcal{H}_{n} S$-locally undetermined for some $S \subseteq\{1, \ldots, n\}$ and $|S|>1$ if there exists a pure state $|\phi\rangle \in \mathcal{H}_{1} \otimes \cdots \otimes \mathcal{H}_{n}$ such that $|\langle\phi \mid \psi\rangle| \neq 1$, and for each $k \in S,|\phi\rangle$ shares the same $(n-1)$-party reduced states with $|\psi\rangle$ when tracing out the $k$ th subsystem, i.e., $\operatorname{Tr}_{k}|\psi\rangle\left\langle\psi\left|=\operatorname{Tr}_{k}\right| \phi\right\rangle\langle\phi| . R_{S}(\psi)$ can be defined similarly. Then all the results presented in this section can be extended to this general notion of $S$-local determinability by simply replacing the index range $\{1, \ldots, n\}$ by $S$. For example, the result corresponding to Theorem 1 can be stated as follows: $|\psi\rangle$ is $S$-locally undetermined if and only if there exist projectors $\left\{P_{j}^{i}: i \in S ; j=1, \ldots, L\right\}, L>1$, such that for any fixed $i \in S, P_{j}^{i}: j=1, \ldots, L$ are pairwise 
orthogonal projectors on $\mathcal{H}_{i}, P_{j}^{i}|\psi\rangle \neq 0$, and $|\psi\rangle=\sum_{j=1}^{L} \bigotimes_{i \in S} P_{j}^{i}|\psi\rangle$. Furthermore, when $|\psi\rangle$ is $S$-locally undetermined, then

$$
R_{S}(\psi)=\left\{\sum_{j=1}^{L} \exp \left(1 \theta_{j}\right) \bigotimes_{i \in S} P_{j}^{i}|\psi\rangle: P_{j}^{i} \text { satisfy the conditions above, and } 0 \leq \theta_{1}, \ldots, \theta_{L}<2 \pi\right\} .
$$

\section{Application in distributed consensus}

The purpose of this section is, similar to that of [11, to characterize the exact quantum resource that is sufficient and necessary to solve distributed consensus problem, by applying the notion of local determinability. As pointed out in Introduction, D'Hondt and Panangaden considered anonymous network setting in which all agents are completely identical without an individual name to distinguish them. As a result, the protocols executed by all agents are the same, and the shared entangled states, as a quantum resource to solve the problem, is invariant under any permutation of agent subspaces. Here in the current paper, however, we relax this constraint to consider more general network setting which is not necessarily anonymous. Interestingly, we find that locally undetermined pure states play a key role in solving distributed consensus for this general network, just like GHZ-like states play in anonymous setting.

Theorem 4 Suppose a set of physically separated agents $A_{1}, \ldots, A_{n}$ share a multipartite pure quantum state $|\psi\rangle \in \mathcal{H}_{1} \otimes \cdots \otimes \mathcal{H}_{n}$ where agent $A_{i}$ holds the particle in $\mathcal{H}_{i}$. Furthermore, communication between them, classical or quantum, is unreliable and local ancillary quantum systems are forbidden. Then there exists a totally correct protocol for these agents to reach a consensus if and only if $|\psi\rangle$ is locally undetermined (equivalently, $|\psi\rangle$ is GSD).

Proof. The sufficiency part is easy from Theorem 1 and Proposition 1 . Suppose $|\psi\rangle$ is locally undetermined. Then there exist projectors $\left\{P_{j}^{i}: i=1, \ldots, n ; j=1, \ldots, L\right\}, L \geq 2$, such that for any fixed $i, P_{j}^{i}: j=1, \ldots, L$ constitute a projective measurement in $\operatorname{supp}\left(\rho_{i}^{\psi}\right)$, and $|\psi\rangle=$ $\sum_{j=1}^{L} \bigotimes_{i=1}^{n} P_{j}^{i}|\psi\rangle$. Let $P_{i}$ be the projector to the ortho-complement of $\operatorname{supp}\left(\rho_{i}^{\psi}\right)$ in $\mathcal{H}_{i}$. Then a simple but totally correct protocol for these $n$ agents to reach a consensus is as follows: agent $i$ performs the projective measurement $\left\{P_{i}, P_{j}^{i}: j=1, \ldots, L\right\}$ on his/her shared particle, and treat the measurement outcome as the agreement they meet. Since the probability of obtaining the outcome corresponding to $P_{i}$ is 0 , and for any $1 \leq j_{1}, \ldots, j_{n} \leq L$,

$$
\bigotimes_{i=1}^{n} P_{j_{i}}^{i}|\psi\rangle=\bigotimes_{i=1}^{n} P_{j_{i}}^{i} \sum_{j=1}^{L} \bigotimes_{i=1}^{n} P_{j}^{i}|\psi\rangle=\sum_{j=1}^{L} \bigotimes_{i=1}^{n} P_{j_{i}}^{i} P_{j}^{i}|\psi\rangle
$$

we deduce that $\bigotimes_{i=1}^{n} P_{j_{i}}^{i}|\psi\rangle \neq 0$ if and only if $j_{1}=\cdots=j_{n}$. That is, the agents will definitely get a common measurement outcome, and so reach a consensus.

For the necessity part, we note that since communication between the agents are unreliable, no classical post-processing is allowed for the protocol to be totally correct. Furthermore, by assumption local ancillary systems in their labs are also forbidden. As a consequence, the only way for them to reach agreement is each performing a projective measurement $\left\{Q_{j}^{i}: \sum_{j} Q_{j}^{i}=I_{\mathcal{H}_{i}}\right\}$ and announcing the outcome as their consensus. Deleting all the projectors $Q_{j}^{i}$ which satisfy $Q_{j}^{i}|\psi\rangle=0$ from $\left\{Q_{j}^{i}\right\}$ we get a set of pairwise orthogonal projectors $P_{j}^{i}: j=1, \ldots, L ; L \geq 2$ such that $\sum_{j=1}^{L} P_{j}^{i} \geq I_{\operatorname{supp}\left(\rho_{i}^{\psi}\right)}$. Then for any $1 \leq j, j^{\prime} \leq L$ and $i \neq i^{\prime}, P_{j}^{i} \otimes P_{j^{\prime}}^{i^{\prime}}|\psi\rangle=\delta_{j, j^{\prime}} P_{j}^{i} \otimes P_{j}^{i^{\prime}}|\psi\rangle$. So we have

$$
P_{j}^{1}|\psi\rangle=\sum_{j^{\prime}=1}^{L} P_{j^{\prime}}^{2} \otimes P_{j}^{1}|\psi\rangle=P_{j}^{2} \otimes P_{j}^{1}|\psi\rangle=\cdots=\bigotimes_{i=1}^{n} P_{j}^{i}|\psi\rangle,
$$


and then

$$
|\psi\rangle=\sum_{j=1}^{L} P_{j}^{1}|\psi\rangle=\sum_{j=1}^{L} \bigotimes_{i=1}^{n} P_{j}^{i}|\psi\rangle .
$$

From Theorem [1, $|\psi\rangle$ is locally undetermined.

\section{Conclusion}

In this paper, we investigate the problem of locally determining multipartite pure states. Necessary and sufficient conditions under which a pure state is locally undetermined among pure states, as well as the precise form of all the pure states sharing the same set of reduced states with it, are presented. As an application, we prove that a locally undetermined pure state can serve as a quantum resource to solve distributed consensus problem in a general network setting. More importantly, such states are the only possible pure states which can achieve this goal in a totally correct and completely fault-tolerant way.

What concerns us in this paper is local determinability of pure state among pure states. There are two natural extensions of this issue: (i) to determine a pure state among all states, pure or mixed; (ii) to determine a mixed state among all states. In fact, Linden et al.'s work [2, 3] is in the framework of (i). New techniques must be proposed to give solutions for these two general problems. Furthermore, to explore properties of multipartite pure entanglement by using the results and techniques developed in this paper is also a direction worthwhile for further study.

\section{Acknowledgement}

The authors thank the colleagues in the Quantum Computation and Quantum Information Research Group for useful discussion. This work was partially supported by the FANEDD under Grant No. 200755, the 863 Project under Grant No. 2006AA01Z102, and the Natural Science Foundation of China (Grant Nos. 60503001, 60621062).

\section{References}

[1] M. Nielsen and I. Chuang. Quantum computation and quantum information. Cambridge university press, 2000.

[2] N. Linden, S. Popescu, and W. K. Wootters. Almost every pure state of three qubits is completely determined by its two-particle reduced density matrices. Physical Review Letters, 89:207901, 2002.

[3] N. Linden and W. K. Wootters. The parts determine the whole in a generic pure quantum state. Physical Review Letters, 89:277906, 2002.

[4] N. S. Jones and N. Linden. Parts of quantum states. Physical Review A, 71:012324, 2005.

[5] L. Diosi. Three-party pure quantum states are determined by two two-party reduced states. Physical Review A, 70:010302, 2004.

[6] S. N. Walck and D. W. Lyons. Only n-qubit greenberger-horne-zeilinger states are undetermined by their reduced density matrices. Physical Review Letters, 100:050501, 2008.

[7] S. N. Walck and D. W. Lyons. The parts determine the whole except for n-qubit greenbergerhorne-zeilinger states,quant-ph/0808.0859. 2008. 
[8] N. A. Lynch. Distributed algorithms. Morgan Kaufman Publishers, 1996.

[9] M. J. Fischer, N. A. Lynch, and M. S. Paterson. Impossibility of distributed consensus with one faulty process. Journal of the ACM, 32(2):374-382, 1985.

[10] G. Bracha and S. Toueg. Asynchronous consensus and broadcast protocols. Journal of the ACM, 32(4):824-840, 1985.

[11] E. D'Hondt and P. Panangaden. The computational power of the w and ghz states. Quantum Information and Computation, 6(2):173-183, 2005.

[12] M. Ben-Or and A. Hassidim. Fast quantum byzantine agreement. In Proceedings of the 37th ACM Symposium on the Theory of Computing (STOC), pages 481-485, Baltimore, Maryland, USA, 2005.

[13] A. J. Coleman. Necessary conditions for n-representability of reduced density matrices. Journal of Mathematical Physics, 13(2):214-222, 1972.

[14] A. Higuchi. On the one-particle reduced density matrices of a pure three-qutrit quantum state, quant-ph/0309186. 2003.

[15] S. Bravyi. Requirements for compatibility between local and multipartite quantum states. Quantum Information and Computation, 4(1):012-026, 2004.

[16] Y. J. Han, Y. S. Zhang, and G. C. Guo. Compatible conditions, entanglement, and invariants. Physical Review A, 70:042309, 2004.

[17] Y. J. Han, Y. S. Zhang, and G. C. Guo. Compatibility relations between the reduced and global density matrices. Physical Review A, 71:052306, 2005. 